\title{
Domain-specific cognitive impairment in patients with COPD and control subjects
}

\author{
This article was published in the following Dove Press journal: \\ International Journal of COPD \\ 19 December 2016 \\ Number of times this article has been viewed
}

\author{
Fiona AHM Cleutjens ${ }^{\prime}$ \\ Frits ME Franssen' \\ Martijn A Spruit \\ Lowie EGW Vanfleteren' \\ Candy Gijsen' \\ Jeanette B Dijkstra ${ }^{2}$ \\ Rudolf WHM Ponds ${ }^{2}$ \\ Emiel FM Wouters ${ }^{1,3}$ \\ Daisy JA Janssen' \\ 'Department of Research and \\ Education, CIRO, Centre of Expertise \\ for Chronic Organ Failure, Horn, \\ ${ }^{2}$ Department of Medical Psychology, \\ Maastricht UMC+/School for Mental \\ Health and Neurosciences (MHeNS), \\ ${ }^{3}$ Department of Respiratory Medicine, \\ Maastricht UMC+, Maastricht, \\ the Netherlands
}

\begin{abstract}
Impaired cognitive function is increasingly recognized in COPD. Yet, the prevalence of cognitive impairment in specific cognitive domains in COPD has been poorly studied. The aim of this cross-sectional observational study was to compare the prevalence of domain-specific cognitive impairment between patients with COPD and non-COPD controls. A neuropsychological assessment was administered in 90 stable COPD patients and 90 non-COPD controls with comparable smoking status, age, and level of education. Six core tests from the Maastricht Aging Study were used to assess general cognitive impairment. By using $Z$-scores, compound scores were constructed for the following domains: psychomotor speed, planning, working memory, verbal memory, and cognitive flexibility. General cognitive impairment and domain-specific cognitive impairment were compared between COPD patients and controls after correction for comorbidities using multivariate linear and logistic regression models. General cognitive impairment was found in $56.7 \%$ of patients with COPD and in $13.3 \%$ of controls. Deficits in the following domains were more often present in patients with COPD after correction for comorbidities: psychomotor speed $(17.8 \%$ vs $3.3 \% ; P<0.001)$, planning $(17.8 \%$ vs $1.1 \%$; $P<0.001)$, and cognitive flexibility ( $43.3 \%$ vs $12.2 \% ; P<0.001)$. General cognitive impairment and impairments in the domains psychomotor speed, planning, and cognitive flexibility affect the COPD patients more than their matched controls.
\end{abstract}

Keywords: cognitive domains, cognitive functioning, cognitive impairment, comorbidities, COPD

\section{Introduction}

Cognitive impairment has been reported in patients with COPD. ${ }^{1-4}$ The prevalence rates of general cognitive impairment in COPD vary widely, ranging from $5.5 \%$ in a large sample of adults with COPD, measured with the Mini-Mental State Examination (MMSE), ${ }^{5}$ up to $77.0 \%$ in patients with both COPD and hypoxemia, measured with multiple cognitive tests. ${ }^{6}$ Increasing age is the most significant determinant of cognitive impairment, ${ }^{7}$ besides a history of tobacco smoking ${ }^{8}$ and a low educational level. ${ }^{9}$ Although the exact pathogenesis of cognitive impairment in COPD remains unknown, vascular-mediated brain pathology, oxidative stress, hypoxemia, systemic inflammation, and comorbidities are proposed pathways to cognitive impairment. ${ }^{1,2,10,11}$

Several domains of cognitive functioning can be distinguished. ${ }^{1}$ Psychomotor speed and information processing assess cognitively demanding information processing tasks, reaction time, and basic manual motor activity. ${ }^{12}$ Memory is the ability to elaborate, store, retrieve, and use information and is defined according to its function, duration, and content. ${ }^{13}$ Executive functioning includes complex cognitive activities such as planning and cognitive flexibility. Planning assesses the ability to set goals and to 
develop appropriate steps or strategies to effectively and efficiently achieve a desired outcome. ${ }^{14}$ Cognitive flexibility assesses the ability to shift concepts and problem-solving strategies. ${ }^{15}$ The importance of examining domain-specific cognition in COPD patients entering pulmonary rehabilitation (PR) is supported by associations between cognitive impairment in specific domains and poor adherence to therapies in HIV-infected adults, ${ }^{16}$ decreased levels of smoking cessation in community-dwelling older persons, ${ }^{17}$ and negative rehabilitation outcomes in elderly patients recovering from a hip fracture. ${ }^{18}$

The prevalence of domain-specific cognitive impairment in COPD patients has been poorly studied, and the prerehabilitation assessment does not include cognitive functioning tests. To date, studies assessing specific cognitive domains ${ }^{1-3}$ lack a clear theoretical and operational definition of domain-specific cognitive impairment and do not provide prevalence rates. Moreover, an adequate control group matched for age, educational level, and smoking history was lacking. ${ }^{3}$

The aim of this study was to compare general and domain-specific cognitive impairments (ie, psychomotor speed, planning, working memory, verbal memory, and cognitive flexibility) between patients with COPD entering PR and non-COPD control subjects with comparable age, educational level, and smoking history. We hypothesized that patients with COPD have a higher prevalence of cognitive impairment compared to controls and that the higher-order cognitive functions, including planning and cognitive flexibility, are more often affected.

\section{Methods}

\section{Study design}

The current study includes cross-sectional data of the COgnitive-PD study, a longitudinal study on neuropsychological functioning in patients with COPD entering PR. The detailed methodology of this study has been published before. ${ }^{19}$ The study is registered in the Dutch Trial Registry (NTR 4215).

\section{Participants}

Based on a previous sample size calculation, the study sample included a total of 90 patients with clinically stable COPD, according to the Global Initiative for Chronic Obstructive Lung Disease (GOLD) 2011 strategic document, ${ }^{20}$ and 90 nonCOPD control participants. Patients were referred for an interdisciplinary three-day assessment in a tertiary referral center in the Netherlands before the start of a comprehensive interdisciplinary inpatient (8 weeks) or outpatient (16 weeks) PR program, in line with the latest American Thoracic Society/European Respiratory Society (ATS/ERS) Statement on PR. Participants were excluded if they had an exacerbation in the past 4 weeks, insufficient comprehension of the Dutch language, or a diagnosis of dementia. Non-COPD control participants were recruited from within the COPD community, and included care partners, family members, or friends of clinical participants, and via advertisements in local newspapers requesting volunteers from the general population. Information regarding smoking status, age, and education was obtained. If possible, the control participant was one-to-one matched with a COPD patient of the COgnitive-PD study population $(\mathrm{n}=183)$ on smoking status (smoker, former smoker, or never smoker), age (standard deviation $[\mathrm{SD}] \leq 10$ years), and educational level $(\mathrm{SD} \leq 1)$ according to the Dutch Standard Classification of Education (CBS). ${ }^{21}$ If not, the control participant was put on a voluntary waiting list to be contacted after inclusion of a COPD patient with matching criteria of the control participant. Exclusion criteria for control participants were as follows: a diagnosis of COPD or asthma, dementia in their medical history, and Dutch language difficulties. All participants gave written informed consent. The Medical Ethics Committee of the University Hospital Maastricht approved this study (NL45127.068.13).

\section{Procedures}

Home visits were performed from November 2013 up to June 2015, between the three-day assessment and the first day of PR, excluding the potential effect of PR on cognitive functioning. The following characteristics were assessed: age, gender, educational level, marital status, visual and hearing impairment, handedness, and intelligence quotient (IQ, by using a shortened version of the Groninger Intelligence Test), ${ }^{22}$ and smoking behavior. The Charlson comorbidity index ${ }^{23}$ was used to quantify self-reported comorbidities. During the three-day assessment, data on body mass index (BMI), functional exercise capacity (measured with two six-minute walk distance [6MWD]) tests according to ERS/ ATS guidelines), ${ }^{24}$ spirometry (forced vital capacity [FVC], $\mathrm{FVC} \%$ predicted, forced expiratory volume in 1 second $\left[\mathrm{FEV}_{1}\right], \mathrm{FEV}_{1} \%$ predicted, and $\mathrm{FEV}_{1} / \mathrm{FVC}$ ), previous selfreported diagnosis of obstructive sleep apnea syndrome (OSAS), neurological disease (eg, cerebrovascular accident, hernia, concussion, migraine, or Parkinson), or psychiatric disease (eg, major depressive disorder, post-traumatic stress disorder, or schizophrenia), physician-diagnosed 
hypertension, and psychological well-being (using the Hospital Anxiety and Depression Scale $[\mathrm{HADS}]^{25}$ and the Beck Depression Inventory $)^{26}$ were collected as part of the clinical routine.

The neuropsychological assessment comprised a detailed testing battery consisting of the following subtests: MMSE, the Stroop Color-Word Test, the Concept Shifting Test, the Letter Digit Substitution Test, the Visual Verbal Learning Task, the Digit Span (DS) from the Wechsler Adult Intelligence Scale III (WAIS-III) and a shortened version of the Behavioral Assessment of the Dysexecutive Syndrome (BADS) including subtasks Key Search and Zoo-map. A validated Dutch translation of the Cognitive Failure Questionnaire (CFQ) was used as a subjective measure of cognitive failure. Subscores, subtests, and parameter measures are described in Table S1. For detailed information, see the section "Supplementary materials".

\section{Statistical analysis}

All analyses were performed using SPSS 21.0 (SPSS Inc., Chicago, IL, USA). A $P$-value $\leq 0.01$ was considered to be statistically significant. Analysis of cognitive performance included descriptive statistics using frequencies for categorical variables, and mean and standard deviation (SD) for continuous variables. Reference data were derived from the Maastricht Aging Study (MAAS), a large Dutch longitudinal study examining the determinants of cognitive aging in a large group of cognitively intact people aged $24-81$ years. ${ }^{27}$ For the DS and BADS, normative data in scoring were used.

Raw scores on neuropsychological tests were transformed into a standardized $Z$-score $(Z=\{x-$ mean $\} / \mathrm{SD})$ based on its mean and SD calculated from the reference group, and were adjusted for age, gender, and educational level. Six core subtests used in MAAS ${ }^{28}$ were used to assess general cognitive impairment (Table S2). General cognitive impairment was defined as a $Z$-score less than $1.0 \mathrm{SD}^{29}$ below the age-, gender-, and education-specific mean of the MAAS study population for at least two of the six core tests. ${ }^{28}$ Individual cognitive measures were grouped into the following specific cognitive domains: psychomotor speed, planning, working memory, verbal memory, and cognitive flexibility (Table S3). Within each domain, scaled $Z$-scores were summed and averaged to yield one compound $Z$-score per cognitive domain. Cognitive impairment in a specific domain was defined as a compound $Z$-score of less than 1.0 SD below the age-, gender-, and education-specific mean of the MAAS study population. ${ }^{29}$ Global cognitive impairment was defined as a score of 24 or below on the MMSE. ${ }^{30}$
A comparison of raw scores and compound $Z$-scores among COPD patients and control participants was made using an independent sample $t$-test or Mann-Whitney $U$-test, as appropriate. Proportions of patients and control participants with general and domain-specific cognitive impairment were calculated and compared with chi-square tests. Multivariate linear or logistic regression models were performed to adjust for the comorbidities, with a significantly higher prevalence rate found in patients than in controls: myocardial infarction, peripheral vascular disease, hemiplegia, clinically relevant symptoms of depression, and clinically relevant symptoms of anxiety. A post hoc analysis was performed in order to compare neuropsychological measures between COPD patients and controls without comorbidities on the Charlson comorbidity index using chi-square tests for categorical variables and independent sample $t$-test or Mann-Whitney $U$-test, as appropriate for continuous variables.

\section{Results}

\section{General characteristics of participants}

The current sample is comparable with the total COgnitive-PD study sample regarding age, gender, lung function, and IQ (all $P>0.05$ ). In addition to a reduced lung function, patients with COPD had a lower functional exercise capacity and IQ, compared to non-COPD controls, while Charlson comorbidity index score was increased. Myocardial infarction, peripheral vascular disease, hemiplegia, and symptoms of anxiety and depression were more often reported by patients with COPD. Moreover, patients with COPD more often reported the use of antidepressants (Table 1).

\section{Prevalence of general cognitive impairment}

According to the MMSE, global cognitive impairment did not differ between the COPD patients and non-COPD controls (Table 2). The prevalence rate of general cognitive impairment, as assessed with the COgnitive-PD neuropsychological assessment, was $56.7 \%$ in the current sample of patients with COPD compared to $13.3 \%$ for controls. Raw mean scores on all core tests were significantly worse in patients with COPD compared to controls (Table 2).

\section{Prevalence of domain-specific cognitive impairment}

The prevalence of cognitive impairment across the five cognitive domains was significantly higher among the included patients with COPD than among controls, except for working memory. Multivariate analyses confirmed a 
Table I Characteristics of the study populations

\begin{tabular}{|c|c|c|c|}
\hline Characteristic & $\begin{array}{l}\text { COPD patients } \\
(n=90)\end{array}$ & $\begin{array}{l}\text { Controls } \\
(n=90)\end{array}$ & $P$-value \\
\hline \multicolumn{4}{|l|}{ Demographics } \\
\hline Age, years, mean (SD) & $63.7(8.8)$ & $62.3(7.4)$ & 0.260 \\
\hline Male, n (\%) & $49(54.4)$ & $45(50.0)$ & 0.327 \\
\hline Lower general or vocational education, $\mathrm{n}(\%)$ & $38(42.2)$ & $25(27.8)$ & 0.187 \\
\hline Married, n (\%) & $54(60.0)$ & $62(68.9)$ & 0.706 \\
\hline \multicolumn{4}{|l|}{ Spirometry } \\
\hline $\mathrm{FEV}_{1} / \mathrm{FVC}$, mean $(\mathrm{SD})^{\mathrm{a}}$ & $41.9(15.1)$ & $77.8(4.7)$ & $<0.001$ \\
\hline $\mathrm{FEV}_{1}\left(\%\right.$ predicted), mean (SD) ${ }^{\mathrm{a}}$ & $54.5(23.7)$ & $116.3(18.2)$ & $<0.001$ \\
\hline \multicolumn{4}{|l|}{ Clinical characteristics } \\
\hline Visual impairment, n (\%) & $17(18.9)$ & II (I2.2) & 0.152 \\
\hline Hearing impairment, $n(\%)$ & $19(21.1)$ & $23(25.6)$ & 0.299 \\
\hline Right-handed by nature, $\mathrm{n}(\%)$ & $72(80.0)$ & $70(77.8)$ & 0.214 \\
\hline $\mathrm{BMI}\left(\mathrm{kg} / \mathrm{m}^{2}\right)$, mean $(\mathrm{SD})$ & $27.3(6.6)$ & $27.3(4.2)$ & 0.987 \\
\hline 6MWD $(\mathrm{m})$, mean $(S D)^{\mathrm{a}}$ & $426.0(102.4)$ & $633.5(96.8)$ & $<0.001$ \\
\hline 6MWD (\% predicted), mean (SD) & $67(15)$ & $95(28)$ & $<0.001$ \\
\hline $\mathrm{PaO}_{2}$, mean (SD) & $9.8(1.6)$ & - & - \\
\hline $\mathrm{PaCO}_{2}$, mean (SD) & $5.1(0.9)$ & - & - \\
\hline Oxygen therapy, n (\%) & $18(20.0)$ & $0(0.0)$ & - \\
\hline $\mathrm{IQ}$, mean $(\mathrm{SD})$ & $84.2(15.8)$ & $99.2(14.6)$ & $<0.001$ \\
\hline Anxiolytics and/or hypnotics, $n$ (\%) & $7(7.8)$ & $\mathrm{I}(\mathrm{I} . \mathrm{I})$ & 0.032 \\
\hline Antidepressants, n (\%) & $26(28.9)$ & $2(2.2)$ & $<0.001$ \\
\hline \multicolumn{4}{|l|}{ Alcohol intake and smoking behavior } \\
\hline$\geq$ I Alcohol units per day, $\mathrm{n}(\%)$ & $30(38.6)$ & - & - \\
\hline Current smoker, n (\%) & $12(13.3)$ & $12(13.3)$ & 1.000 \\
\hline Former smoker, $\mathrm{n}(\%)$ & $75(83.3)$ & $75(83.3)$ & \\
\hline Never smoker, n (\%) & $3(3.3)$ & $3(3.3)$ & \\
\hline \multicolumn{4}{|l|}{ Comorbidities } \\
\hline Charlson comorbidity index score, points, mean (SD) & $3.0(1.8)$ & $0.9(1.5)$ & $<0.001$ \\
\hline Myocardial infarction, $\mathrm{n}(\%)$ & $16(17.8)$ & $5(5.6)$ & 0.009 \\
\hline Congestive heart failure, $\mathrm{n}(\%)$ & $10(11.1)$ & $2(2.2)$ & 0.016 \\
\hline Peripheral vascular disease, $\mathrm{n}(\%)$ & $23(25.6)$ & $9(10.0)$ & 0.005 \\
\hline Cerebrovascular disease, $\mathrm{n}(\%)$ & $11(12.2)$ & $4(4.4)$ & 0.052 \\
\hline Connective tissue disease, $\mathrm{n}(\%)$ & $14(15.6)$ & $16(17.8)$ & 0.421 \\
\hline Peptic ulcer disease, $\mathrm{n}(\%)$ & $11(12.2)$ & $6(6.7)$ & 0.154 \\
\hline Mild, moderate, or severe liver disease, $n(\%)$ & $2(2.2)$ & $\mathrm{I}(\mathrm{l} . \mathrm{I})$ & 0.500 \\
\hline Diabetes mellitus, $\mathrm{n}(\%)$ & $15(16.7)$ & $10(11.1)$ & 0.195 \\
\hline Hemiplegia, $n(\%)$ & $10(11.1)$ & $I(I . I)$ & 0.005 \\
\hline Moderate-to-severe chronic kidney disease, n (\%) & $4(4.4)$ & $0(0.0)$ & 0.341 \\
\hline Solid or malignant tumors, $n(\%)$ & $15(16.7)$ & $7(7.8)$ & 0.055 \\
\hline Hypertension, n (\%) & $70(77.8)$ & - & - \\
\hline OSAS, n (\%) & $13(14.4)$ & $7(7.8)$ & 0.118 \\
\hline Neurological disease, $n(\%)$ & $20(22.0)$ & $9(10.0)$ & 0.021 \\
\hline Psychiatric disease, $\mathrm{n}(\%)$ & II (I2.2) & $4(4.4)$ & 0.052 \\
\hline \multicolumn{4}{|l|}{ Psychological well-being } \\
\hline HADS anxiety score (points), mean (SD) ${ }^{\mathrm{a}}$ & $8.3(4.5)$ & $3.9(3.1)$ & $<0.001$ \\
\hline HADS anxiety score $>10$ points, $n(\%)$ & $28(31.1)$ & $5(5.6)$ & $<0.001$ \\
\hline HADS depression score (points), mean (SD) ${ }^{a}$ & $7.7(3.9)$ & $2.2(2.5)$ & $<0.001$ \\
\hline HADS depression score $>10$ points, $n(\%)$ & $19(21.1)$ & $2(2.2)$ & $<0.001$ \\
\hline BDI score, points, mean $(S D)^{\mathrm{a}}$ & $16.3(8.8)$ & $5.1(5.9)$ & $<0.001$ \\
\hline
\end{tabular}

Notes: aNonparametric statistical tests have been used because of skewed data; '-' indicates no data.

Abbreviations: BDI, Beck Depression Inventory; BMI, body mass index; FEV , forced expiratory volume in I second; FVC, forced vital capacity; HADS, Hospital Anxiety and Depression Scale; IQ, intelligence quotient; OSAS, obstructive sleep apnea syndrome; $\mathrm{PaO}_{2}$, arterial partial pressure of oxygen; $\mathrm{PaCO}_{2}$, arterial partial pressure of carbon dioxide; SD, standard deviation; 6MWD, 6-minute walk distance.

higher prevalence of impairments across all domains, except for working memory (Figure 1). Results remained the same when the matching variables age, education, and smoking status, in addition to the comorbidities, were included in the multivariate model (Table S4). Patients with and without oxygen therapy did not significantly differ on general cognitive impairment or any of the cognitive domains (all $P$-values $>0.01$; Table $\mathrm{S} 5$ ). The same was true when comparing COPD patients with clinically relevant symptoms of depression or anxiety (HADS depression or anxiety 
Table 2 Raw unadjusted mean values on neuropsychological measures and compound Z-scores in patients with COPD and controls

\begin{tabular}{|c|c|c|c|c|}
\hline Outcome measure & $\begin{array}{l}\text { COPD patients } \\
(n=90)\end{array}$ & $\begin{array}{l}\text { Controls } \\
(n=90)\end{array}$ & $P$-value & Adjusted $P$-value ${ }^{a}$ \\
\hline \multicolumn{5}{|l|}{ Global cognitive functioning } \\
\hline MMSE, mean $(S D)^{b}$ & $27.4(2.3)$ & $28.1(1.6)$ & 0.017 & 0.016 \\
\hline Global cognitive impairment (MMSE $\leq 24), n(\%)$ & $7(7.8)$ & $2(2.2)$ & 0.084 & 0.243 \\
\hline \multicolumn{5}{|l|}{ Domain-specific cognition } \\
\hline Psychomotor speed (compound Z-score), mean (SD) & $-0.5(1.1)$ & $0.3(0.7)$ & $<0.001$ & $<0.001$ \\
\hline SCWT card I, mean (SD) ${ }^{\mathrm{b}}$ & $19.1(5.6)$ & $17.0(2.6)$ & 0.003 & 0.010 \\
\hline CST-A, mean (SD) & $28.1(15.8)$ & $21.2(5.6)$ & $<0.001$ & $<0.001$ \\
\hline LDST 60 second written, mean (SD) & $25.2(7.2)$ & $31.7(5.7)$ & $<0.001$ & $<0.001$ \\
\hline LDST 60 second oral, mean (SD) & $3 \mathrm{I} .1(7.5)$ & $37.1(6.5)$ & $<0.00$ I & $<0.001$ \\
\hline Planning (compound Z-score), mean (SD) & $-0.3(0.8)$ & $0.3(0.6)$ & $<0.001$ & $<0.001$ \\
\hline BADS key search, mean (SD) & $2.2(1.5)$ & $3.1(1.2)$ & $<0.001$ & 0.002 \\
\hline BADS zoo map, mean (SD) ${ }^{\mathrm{b}}$ & $2.2(0.8)$ & $2.7(0.7)$ & $<0.001$ & $<0.001$ \\
\hline Working memory (compound Z-score), mean (SD) & $-0.6(0.8)$ & $-0.2(0.8)$ & 0.004 & 0.035 \\
\hline VVLT trial I, mean (SD) & $4.4(1.9)$ & $5.5(2.1)$ & $<0.001$ & 0.001 \\
\hline DS backward, mean (SD) & $3.0(1.1)$ & $3.1(1.2)$ & 0.556 & 0.961 \\
\hline Verbal memory (compound Z-score), mean (SD) & $-0.5(1.1)$ & $0.0(1.0)$ & 0.001 & 0.002 \\
\hline VVLT total recall I-5, mean (SD) & $40.2(10.8)$ & $47.3(10.2)$ & $<0.001$ & $<0.001$ \\
\hline VVLT delayed recall, mean (SD) & $7.3(3.5)$ & $9.1(3.2)$ & 0.001 & 0.002 \\
\hline VVLT retention max, mean $(S D)^{b}$ & $0.6(0.3)$ & $0.7(0.2)$ & 0.020 & 0.030 \\
\hline Cognitive flexibility (compound Z-score), mean (SD) & $-1.2(1.6)$ & $-0.0(0.8)$ & $<0.001$ & $<0.001$ \\
\hline SCWT card III, mean (SD) ${ }^{\mathrm{b}}$ & $60.9(26.7)$ & $43.0(13.0)$ & $<0.001$ & $<0.001$ \\
\hline CST-C, mean (SD) & $51.1(26.3)$ & $35.3(11.6)$ & $<0.001$ & $<0.001$ \\
\hline General cognitive impairment ( 2 out of 6 subtest & $5 \mathrm{I}(56.7)$ & $12(13.3)$ & $<0.001$ & $<0.001$ \\
\hline \multicolumn{5}{|l|}{ Z-scores - I SD), n (\%) } \\
\hline \multicolumn{5}{|l|}{ Cognitive complaints } \\
\hline CFQ absentmindedness, mean (SD) & $8.2(5.4)$ & $7.1(3.6)$ & 0.112 & 0.619 \\
\hline CFQ social interactions, mean (SD) & $5.7(3.3)$ & $4.9(2.2)$ & 0.042 & 0.855 \\
\hline CFQ names and words, mean (SD) & $5.7(2.6)$ & $4.9(2.0)$ & 0.021 & $0.08 \mathrm{I}$ \\
\hline CFQ orientation, mean (SD) & $2.7(2.6)$ & $2.3(1.7)$ & 0.352 & 0.638 \\
\hline CFQ total score, mean (SD) & $31.8(17.3)$ & $27.3(11.0)$ & 0.038 & 0.987 \\
\hline
\end{tabular}

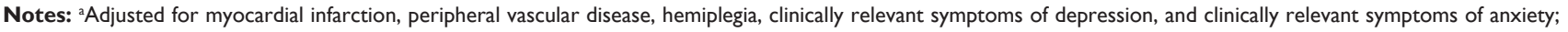
bnonparametric statistical tests have been used because of skewed data.

Abbreviations: BADS, Behavioral Assessment of the Dysexecutive Syndrome; CFQ, Cognitive Failure Questionnaire; CST-A, Concept Shifting Test part A; CST-C, Concept Shifting Test part C; DS, Digit Span; LDST, Letter Digit Substitution Test; MMSE, Mini-Mental State Examination; SCWT, Stroop Color-Word Test; SD, standard deviation; VVLT, Visual Verbal Learning Test.

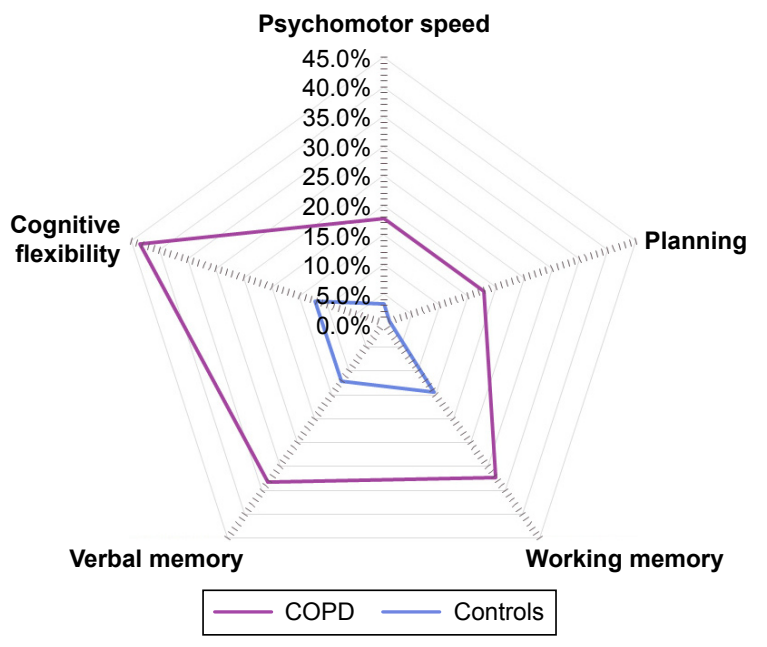

Figure I Domain-specific cognitive impairment after correcting for myocardial infarction, peripheral vascular disease, hemiplegia, clinically relevant symptoms of depression, and clinically relevant symptoms of anxiety. Proportion of patients with COPD and controls with impairments in the domains psychomotor speed $(17.8 \%$ vs $3.3 \% ; P<0.001)$, planning ( $17.8 \%$ vs $1.1 \% ; P<0.00 I)$, working memory $(32.3 \%$ vs $14.4 \% ; P=0.035$ ), verbal memory ( $33.3 \%$ vs $12.2 \% ; P=0.002$ ), and cognitive flexibility (43.3\% vs I2.2\%; $P<0.00 I)$. score $>10$ points) and those without clinically relevant symptoms of depression or anxiety (all $P$-values $>0.01$; $\underline{\text { Tables } \mathrm{S} 6}$ and $\underline{\mathrm{S} 7}$ ).

\section{Cognitive complaints}

The CFQ total score and subscores did not differ between patients with COPD and non-COPD controls (Table 2). These results were confirmed by multivariate analyses.

\section{Cognitive impairment in COPD patients and controls without comorbidities}

In total, 18 patients with COPD reported no disease other than COPD on the Charlson index, and 43 non-COPD controls reported no comorbidities on the Charlson index and were included in a post hoc analysis (Table 3). The prevalence of general cognitive impairment did not differ significantly between patients and controls without comorbidities $(50 \%$ and $18.6 \%$, respectively). Global cognitive impairment, 
Table 3 Characteristics of the selected study population without comorbidities

\begin{tabular}{|c|c|c|c|}
\hline Characteristic & $\begin{array}{l}\text { COPD patients } \\
(n=18)\end{array}$ & $\begin{array}{l}\text { Controls } \\
(n=43)\end{array}$ & $P$-value \\
\hline \multicolumn{4}{|l|}{ Demographics } \\
\hline Age, years, mean (SD) & $61.9(8.8)$ & $60.3(7.5)$ & 0.477 \\
\hline Male, $\mathrm{n}(\%)$ & $9(50.0)$ & $22(51.2)$ & 0.578 \\
\hline Lower general or vocational education, $\mathrm{n}(\%)$ & $7(38.9)$ & $10(23.3)$ & 0.176 \\
\hline Married, n (\%) & $7(38.9)$ & $34(79.1)$ & 0.003 \\
\hline \multicolumn{4}{|l|}{ Spirometry } \\
\hline $\mathrm{FEV}_{\mathrm{I}} / \mathrm{FVC}$, mean (SD) & $42.1(13.3)$ & $77.3(4.6)$ & $<0.001$ \\
\hline $\mathrm{FEV}_{1}(\%$ predicted), mean (SD) & $59.3(28.0)$ & I I $3.8(10.7)$ & $<0.001$ \\
\hline \multicolumn{4}{|l|}{ Clinical characteristics } \\
\hline Visual impairment, n (\%) & $3(16.7)$ & I (2.3) & 0.073 \\
\hline Hearing impairment, n (\%) & $2(I I . I)$ & $13(30.2)$ & 0.101 \\
\hline Right-handed by nature, $\mathrm{n}(\%)$ & II (6I.I) & $33(76.7)$ & 0.236 \\
\hline BMI $\left(\mathrm{kg} / \mathrm{m}^{2}\right)$, mean $(\mathrm{SD})$ & $25.3(6.5)$ & $27.0(4.2)$ & 0.219 \\
\hline 6MWD $(\mathrm{m})$, mean $(\mathrm{SD})$ & $459.0(131.20)$ & $661.1(106.8)$ & $<0.001$ \\
\hline 6MWD (\% predicted), mean (SD) ${ }^{\mathrm{a}}$ & $71.4(18.3)$ & $94.2(39.0)$ & $<0.001$ \\
\hline $\mathrm{PaO}_{2}$, mean (SD) & I0.I (I.6) & - & - \\
\hline $\mathrm{PaCO}_{2}$, mean $(\mathrm{SD})^{\mathrm{a}}$ & $5.1(0.6)$ & - & - \\
\hline Oxygen therapy, n (\%) & $2(I I . I)$ & - & - \\
\hline $\mathrm{IQ}$, mean $(\mathrm{SD})$ & $84.6(13.9)$ & $100.9(15.6)$ & $<0.001$ \\
\hline Hypertension, n (\%) & $2(I 1.1)$ & - & - \\
\hline OSAS, n (\%) & $0(0.0)$ & $4(9.3)$ & - \\
\hline \multicolumn{4}{|l|}{ Alcohol intake and smoking behavior } \\
\hline$\geq$ I Alcohol units per day, $\mathrm{n}(\%)^{\mathrm{b}}$ & $8(47.1)$ & - & - \\
\hline Current smoker, n (\%) & $2(11.1)$ & $6(14.0)$ & 0.606 \\
\hline Former smoker, n (\%) & $16(88.9)$ & $35(81.4)$ & \\
\hline Never smoker, n (\%) & $0(0.0)$ & $2(4.7)$ & \\
\hline \multicolumn{4}{|l|}{ Psychological well-being } \\
\hline HADS anxiety score (points), mean (SD) ${ }^{\mathrm{a}}$ & $8.4(4.5)$ & $3.4(2.5)$ & $<0.001$ \\
\hline HADS anxiety score $>8$ points, $n$ (\%) & $7(38.9)$ & $\mathrm{I}(2.3)$ & $<0.001$ \\
\hline HADS depression score (points), mean (SD) & $7.5(4.7)$ & $\mathrm{I} .7(\mathrm{l} .8)$ & $<0.001$ \\
\hline HADS depression score $>8$ points, $n$ (\%) & $8(44.4)$ & $0(0.0)$ & $<0.001$ \\
\hline BDI score (points), mean (SD) ${ }^{\mathrm{a}}$ & I7.8 (11.4) & $3.9(4.0)$ & $<0.001$ \\
\hline
\end{tabular}

Notes: aNonparametric statistical tests have been used because of skewed data; ' $\mathrm{n}=$ I7; '-' indicates no data.

Abbreviations: BDI, Beck Depression Inventory; BMI, body mass index; FEV , forced expiratory volume in I second; FVC, forced vital capacity; HADS, Hospital Anxiety and Depression Scale; IQ, intelligence quotient; OSAS, obstructive sleep apnea syndrome; $\mathrm{PaO}_{2}$, arterial partial pressure of oxygen; PaCO ${ }_{2}$, arterial partial pressure of carbon dioxide; SD, standard deviation; 6MWD, 6-minute walk distance.

general cognitive impairment, and cognitive impairment in the domains psychomotor speed, working memory, and verbal memory did not differ between both groups. Patients with COPD more often reported complaints on the CFQ subscale words and names (Table 4). The prevalence of cognitive impairment was higher for patients with COPD without comorbidities than for controls without comorbidities in the domains planning and cognitive flexibility (Figure 2).

\section{Discussion}

\section{Key findings}

This cross-sectional observational study showed a prevalence rate of $56.7 \%$ for cognitive impairment in a cohort of patients with COPD referred for PR by applying a comprehensive neuropsychological assessment. This prevalence was four times higher compared to matched non-COPD controls. Moreover, cognitive impairment in the domains planning and cognitive flexibility was more prevalent in patients with COPD than controls after correction for comorbidities.

\section{Prevalence of cognitive impairment}

Differences in study populations (such as level of hypoxemia and severity of COPD), methodology, sample size, the use of different definitions, and diagnostic criteria for cognitive impairment may explain the wide range of prevalence rates of cognitive impairment in COPD. ${ }^{5,6,31}$ We observed a prevalence rate of $56.7 \%$ for general cognitive impairment, which is within the range of previous studies. In accordance with Singh et al $^{29} Z$-scores below $-1.0 \mathrm{SD}$ were considered as being impaired. The relatively low $Z$-score cutoff of 
Table 4 Raw unadjusted mean values on neuropsychological measures and compound Z-scores in patients with COPD and controls without comorbidities

\begin{tabular}{|c|c|c|c|}
\hline Outcome measure & $\begin{array}{l}\text { COPD patients } \\
(n=18)\end{array}$ & $\begin{array}{l}\text { Controls } \\
(n=43)\end{array}$ & $P$-value \\
\hline \multicolumn{4}{|l|}{ Global cognitive functioning } \\
\hline MMSE, mean (SD) & $28.1(1.4)$ & $28.1(1.3)$ & 0.824 \\
\hline Global cognitive impairment (MMSE $\leq 24), n(\%)$ & $0.0(0.0)$ & $0.0(0.0)$ & - \\
\hline \multicolumn{4}{|l|}{ Domain-specific cognition } \\
\hline Psychomotor speed (compound Z-score), mean (SD) & $-0.2(1.0)$ & $0.3(0.5)$ & 0.039 \\
\hline SCWT card I, mean (SD) & $18.4(4.0)$ & $16.9(2.3)$ & 0.079 \\
\hline CST-A, mean (SD) & $24.7(8.2)$ & $20.5(5.3)$ & 0.019 \\
\hline LDST 60 second written, mean (SD) & $28.4(7.4)$ & $31.8(5.1)$ & 0.044 \\
\hline LDST 60 second oral, mean (SD) & $32.3(6.8)$ & $37.1(6.2)$ & 0.011 \\
\hline Planning (compound Z-score), mean (SD) & $-0.4(0.9)$ & $0.4(0.5)$ & $<0.001$ \\
\hline BADS key search, mean (SD) & $1.9(\mathrm{I} .4)$ & $3.1(1.0)$ & 0.002 \\
\hline BADS zoo map, mean (SD) & $2.2(1.2)$ & $2.8(0.6)$ & 0.042 \\
\hline Working memory (compound Z-score), mean (SD) & $-0.6(0.7)$ & $-0.3(0.8)$ & 0.140 \\
\hline VVLT trial I, mean (SD) & $4.7(2.3)$ & $5.4(2.0)$ & 0.218 \\
\hline DS backward, mean (SD) & $2.8(1.3)$ & $3.1(1.1)$ & 0.262 \\
\hline Verbal memory (compound Z-score), mean (SD) & $-0.5(1.2)$ & $-0.0(1.1)$ & 0.153 \\
\hline VVLT total recall I-5, mean (SD) & $40.5(11.7)$ & $46.7(10.6)$ & 0.049 \\
\hline VVLT delayed recall, mean (SD) & $7.6(3.7)$ & $8.9(3.3)$ & 0.155 \\
\hline VVLT retention max, mean $(S D)^{a}$ & $0.7(0.2)$ & $0.7(0.2)$ & 0.716 \\
\hline Cognitive flexibility (compound Z-score), mean (SD) & $-1.1(1.5)$ & $-0.2(0.7)$ & $<0.001$ \\
\hline SCWT card III, mean (SD) ${ }^{\mathrm{a}}$ & $56.3(15.9)$ & $42.2(12.3)$ & 0.001 \\
\hline CST-C, mean (SD) ${ }^{\mathrm{a}}$ & $48.7(24.3)$ & $33.6(11.2)$ & 0.029 \\
\hline General cognitive impairment ( 2 out of 6 subtest $Z$-scores $-I$ SD), n (\%) & $9(50.0)$ & $8(18.6)$ & 0.016 \\
\hline \multicolumn{4}{|l|}{ Cognitive complaints } \\
\hline CFQ absentmindedness, mean (SD) & $7.2(4.0)$ & $6.5(3.4)$ & 0.480 \\
\hline CFQ social interactions, mean (SD) & $5.8(2.4)$ & $4.6(2.1)$ & 0.044 \\
\hline CFQ names and words, mean (SD) & $6.1(2.1)$ & $4.4(2.0)$ & 0.005 \\
\hline CFQ orientation, mean (SD) & $2.5(2.3)$ & $2.3(1.9)$ & 0.662 \\
\hline CFQ total score, mean (SD) & $30.6(10.9)$ & $25.4(10.5)$ & 0.084 \\
\hline
\end{tabular}

Notes: 'Nonparametric statistical tests have been used because of skewed data. '-' indicates a $p$-value cannot be calculated since both patients and controls do not meet the criteria for global cognitive impairment.

Abbreviations: BADS, Behavioral Assessment of the Dysexecutive Syndrome; CFQ, Cognitive Failure Questionnaire; CST, Concept Shifting Test; DS, Digit Span; LDST, Letter Digit Substitution Test; MMSE, Mini-Mental State Examination; SCWT, Stroop Color-Word Test; SD, standard deviation; VVLT, Visual Verbal Learning Test.

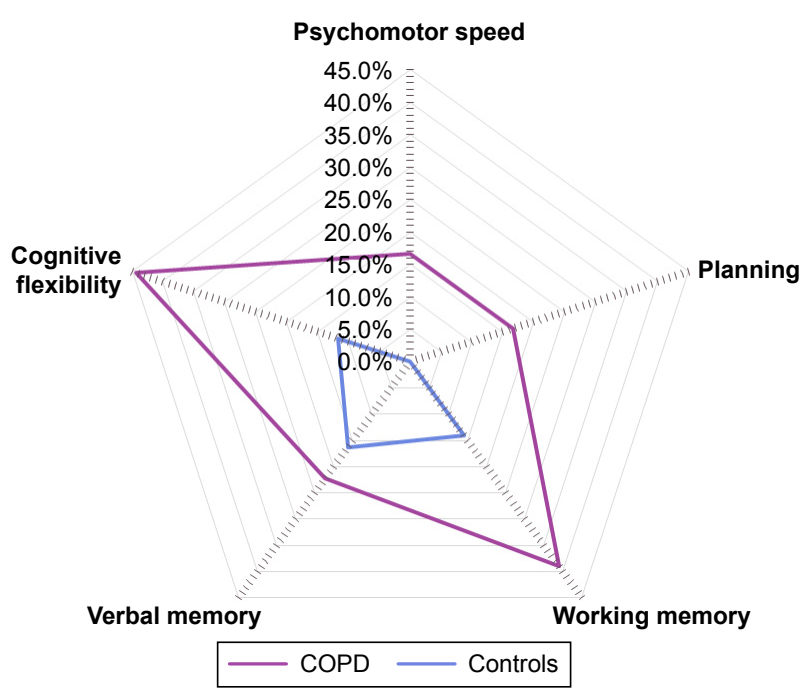

Figure 2 Domain-specific cognitive impairment in patients with COPD and controls without comorbidities. Proportion of patients with COPD and controls with impairments in the domains psychomotor speed ( $16.7 \%$ vs $0.0 \%$; $P=0.039$;), planning $(16.7 \%$ vs $0.0 \% ; P<0.00 I)$, working memory $(38.9 \%$ vs $I 4.0 \% ; P=0.140)$, verbal memory (22.2\% vs $16.3 \% ; P=0.262$ ), and cognitive flexibility ( $44.4 \%$ vs $I I .6 \% ; P<0.00 \mathrm{I}$ ).
-1.0 SD may explain why impairments in some non-COPD controls were present as well. Nevertheless, the use of a control group enhances our understanding of the cognitive profile for patients with COPD by showing that general cognitive impairment is four times more common in patients with COPD compared to controls and that domain-specific cognitive impairment occurs two to sixteen times more often in patients with COPD compared to controls. These differences are less clear when only patients and controls without comorbidities are analyzed. Indeed, abnormal planning was observed in $16.7 \%$ of patients without comorbidities but in none of the controls without comorbidities, and abnormal cognitive flexibility was observed in $44.4 \%$ and $11.6 \%$ of patients and controls without comorbidities, respectively.

Global cognitive impairment, as assessed with the MMSE, did not differ between patients with COPD (7.8\%) and non-COPD controls $(2.2 \%)$. Moreover, the prevalence rate of global cognitive impairment is lower compared to that 
of general cognitive impairment. Both can be explained by methodological factors. The six core tests to assess general cognitive impairment might be more sensitive to detect relatively small impairments in specific areas of cognitive functioning that together may not lead to poor performance on a screening test such as the MMSE. In fact, the MMSE is used to detect dementia, which was an exclusion criterion in the current study, but is not sensitive enough to detect mild cognitive deficits.

\section{Domain-specific cognitive impairment}

Cognitive domains that have been shown to be affected in patients with COPD are memory, learning, attention, concentration, verbal and letter fluency, information processing speed, coordination, intelligence, and mental and cognitive flexibility. ${ }^{3,32}$ In the current study, one out of five patients with COPD showed impairments in the domain psychomotor speed. This may explain clinical features such as a decreased ability to comprehend lengthy, detailed, and fast instructions during therapies or to engage in educational sessions without losing attention. Second, two out of five patients with COPD were impaired in the domain planning. Owing to the inability to transfer previous knowledge to new events, patients with COPD may be hampered in their self-management because of difficulties in handling new problems and situations, and initiating any kind of health behavior change, which is the key component for effective self-management of patients with COPD. ${ }^{33}$ Third, over $30.0 \%$ of patients with COPD showed working memory impairments, which consequently may lead to problems with maintaining given educational information. Fourth, one out of three patients with COPD showed impairments in the domain verbal memory. Patients may seem unmotivated if they forget or ignore given guidelines, requests, or instructions. These clinical features may be a consequence of verbal memory impairment. Finally, over $40 \%$ of patients with COPD showed impairments in the domain cognitive flexibility. These may be related to difficulties in flexible thinking, alternating attention without losing focus, choosing appropriate behavioral responses according to the situation, and initiating behavioral and motor plans and activity, which all together may hamper attempts to adapt lifestyle (eg, quit smoking and become physically active). ${ }^{14,15}$

\section{The role of comorbidities}

After exclusion of patients and controls with comorbidities, differences in the domains psychomotor speed, working memory, and verbal memory between patients and controls waned. COPD is a multimorbid disease,,$^{34,35}$ and it is likely that selected comorbidities explain part of the cognitive impairment in COPD. For example, myocardial infarction and hemiplegia may lead to hypoxia-induced brain cell death and possible cognitive deficits. ${ }^{36}$ Moreover, symptoms of depression and anxiety were increased in patients with COPD, which might explain part of the effect on memory. ${ }^{37}$ Nevertheless, in the current study, we did not find differences in general or domain-specific cognitive impairment when comparing COPD patients with and without symptoms of depression or anxiety. This might be explained by the fact that we used a generic scale to assess symptoms of anxiety and depression instead of a structured psychiatric interview to diagnose a major depressive disorder or anxiety disorder. It is possible that COPD patients with and without a major depressive disorder differ in their cognitive performances. Indeed, deficits in cognitive performances are found in major depressive disorders. ${ }^{38}$ Moreover, the higher prevalence of antidepressant use may partly account for the higher prevalence of cognitive impairment in patients compared to controls due to its sedative and anticholinergic effects. The dose, time since last administration, and plasma concentration of the drug, however, determine the extent to which cognitive performance is affected. ${ }^{39}$

A post hoc analysis in which we compared neuropsychological measures between COPD patients and controls without any comorbidities on the Charlson index revealed that the domains cognitive flexibility and planning were more often affected in patients with COPD. Despite a difference in group sizes between patients and controls, which might distort the results, our results are in line with the idea that higher executive functions are more related to COPD-specific factors, compared to lower cognitive functions. Since hypertension and OSAS, both of which impact cognition and are common in COPD, are not integrated in the Charlson index, these comorbidities may also account for a higher prevalence of cognitive impairment in COPD patients compared to controls. Moreover, $9 \%$ of the total COPD study population without a diagnosis of diabetes on the Charlson comorbidity index had a blood glucose level of $7 \mathrm{mmol} / \mathrm{L}$ or above, and $47 \%$ of patients without physician-diagnosed hypertension had an elevated systolic blood pressure ( $\geq 140 \mathrm{mmHg}$ ) or elevated diastolic blood pressure of ( $\geq 90 \mathrm{mmHg}$ ) and were referred for further diagnosis. These underreported comorbidities may also partly account for the high prevalence of cognitive impairment in COPD. COPD patients had significantly lower 6MWD and IQ scores compared to non-COPD controls, which may also explain impairments in complex, higher-order cognitive functions (eg, executive functioning) ${ }^{40,41}$ Physical 
exercise has been suggested to enhance cognitive vitality of older adults, and therefore it is possible that patients with a lower baseline physical exercise pattern have lower cognitive performances. ${ }^{40}$ Yet, we cannot identify causal relationships, and it remains difficult to draw safe conclusions about the reasons for the presence of domain-specific cognitive impairments in patients with COPD.

\section{Clinical consequences}

Patients with cognitive impairment may be labeled as unmotivated and uncooperative because of problems with treatment adherence, treatment attrition, or negative treatment outcomes. Moreover, patients with COPD may be stigmatized as resistant, unmotivated, and in denial. ${ }^{42}$ However, rather than misattributing such problems as poor motivation to a character trait, cognitive impairment may be the culprit. For example, a recent study shows that cognitive functioning as assessed with the Montreal Cognitive Assessment is able to distinguish COPD patients with poor adherence to inhaler therapy from those with frequent errors in inhaler use technique. ${ }^{43}$ Therefore, a comprehensive assessment of cognitive functioning and accurately distinguishing cognitive impairment among multiple cognitive domains might become more important, as more specific therapy interventions can be developed by taking into account the possible consequences of domain-specific cognitive impairment. Moreover, in patients with brain injuries, Alzheimer disease, and multiple sclerosis, cognitive training strategies, which aim to improve cognitive functioning and consequently reduce functional and psychological problems, have already been applied. ${ }^{44,45}$ To our knowledge, no study has been conducted to explore the effect of cognitive training strategies on cognitive performance in patients with COPD.

Even though we demonstrated that cognitive impairment is more common in patients with COPD compared to controls, subjectively reported memory complaints on the CFQ were within the normal range of $21-43$ in both groups. ${ }^{46}$ It is possible that impairments in cognition may gradually grow into deficits so that they are not easily recognized by the patient. Moreover, the burden of COPD may outflank the seriousness of self-experienced cognitive impairments and hence the recognition of daily cognitive difficulties.

\section{Future research}

The high prevalence of general and domain-specific cognitive impairment in our study population raises questions about its consequences. Longitudinal follow-up studies are needed to assess whether cognitive impairment in specific domains affects the educational components, behavior change interventions, and other outcomes of PR. Moreover, it might be worthwhile to assess whether cognitive training strategies are able to slow down or prevent cognitive decline, or whether it has a beneficial effect on a PR trajectory and its sustained effects.

\section{Methodological considerations}

By using an extensive neuropsychological assessment, we were able to estimate the prevalence of general and domainspecific cognitive impairment in patients with COPD and matched controls. The major determinants of cognitive impairment include a history of cigarette smoking, increasing age, and educational level..$^{7-9}$ Therefore, the inclusion of a matched comparison group, a major strength of this study, might rule out the effect of smoking, age, and education on cognitive functioning. Although we did not match for gender and IQ, there were no significant differences in gender between both groups, and $Z$-scores were adjusted for educational level, age, and gender. Despite these strengths, this study has some limitations. Patients and controls were not matched for comorbidities, and data on arterial blood gases, alcohol intake, and hypertension in controls were not available. However, a post hoc analysis was performed in order to compare cognitive functioning between patients and controls without comorbidities. The sample size of the post hoc analysis was small, and therefore this analysis might be underpowered. However, extrapulmonary comorbidities are common and significant in COPD, and therefore our study sample is representative of the COPD population. Yet, we included patients from a tertiary referral center, which may limit the generalizability of the results because, in general, patients with more advanced COPD are being referred. Nevertheless, these data are of significant importance for COPD patients who follow a PR program. Because of multiple comparisons and to decrease the number of false negatives, a $P$-value $\leq 0.01$ was used instead of the commonly used $P$-value of $\leq 0.05$. Also, selection bias may reduce the generalizability of our results. Patients and controls with subjective complaints of cognitive functioning might avoid participation due to anxiety or worry, or may have been more willing to participate in the present study due to personal interest.

\section{Conclusion}

The results of this study underline that general cognitive impairment and cognitive impairment in specific domains, namely psychomotor speed, planning, and cognitive flexibility, affect a higher proportion of patients with COPD 
than their matched controls. Comorbid diseases in COPD are likely to affect cognitive performance in COPD. Yet, impairments in executive functions may be more related to COPD-specific factors. The identification of domain-specific cognitive impairment is necessary for further optimizing therapies (eg, smoking cessation, self-management programs, and PR) for patients with COPD. Longitudinal follow-up of our participants will enable us to examine the effects of cognitive impairment in specific domains on PR outcomes, such as functional status, health status, psychological wellbeing, and the patient's knowledge about COPD and their need for information.

\section{Acknowledgments}

The authors would like to thank all subjects for their participation. They are also grateful to the staff of the biometry and lung function laboratory at CIRO, Horn, for their help with assessing the participants.

\section{Disclosure}

The authors report no conflicts of interest in this work.

\section{References}

1. Cleutjens FA, Janssen DJ, Ponds RW, Dijkstra JB, Wouters EF. COgnitive-pulmonary disease. BioMed Res Int. 2014;2014:697825.

2. Dodd JW, Getov SV, Jones PW. Cognitive function in COPD. Eur Respir J. 2010;35(4):913-922.

3. Schou L, Østergaard B, Rasmussen LS, Rydahl-Hansen S, Phanareth K. Cognitive dysfunction in patients with chronic obstructive pulmonary disease - a systematic review. Respir Med. 2012;106(8): 1071-1081.

4. Shrikrishna D, Hopkinson NS. Chronic obstructive pulmonary disease: consequences beyond the lung. Clin Med (Lond). 2012;12(1):71-74.

5. Thakur N, Blanc PD, Julian LJ, et al. COPD and cognitive impairment: the role of hypoxemia and oxygen therapy. Int J Chron Obstruc Pulmon Dis. 2010;5:263-269.

6. Grant I, Heaton RK, McSweeny AJ, Adams KM, Timms RM. Neuropsychologic findings in hypoxemic chronic obstructive pulmonary disease. Arch Intern Med. 1982;142(8):1470-1476.

7. Barnes JN. Exercise, cognitive function, and aging. Adv Physiol Educ. 2015;39(2):55-62.

8. Anstey KJ, von Sanden C, Salim A, O'Kearney R. Smoking as a risk factor for dementia and cognitive decline: a meta-analysis of prospective studies. Ame J Epidemiol. 2007;166(4):367-378.

9. Evans DA, Beckett LA, Albert MS, et al. Level of education and change in cognitive function in a community population of older persons. Ann Epidemiol. 1993;3(1):71-77.

10. Cleutjens FA, Janssen DJ, Gijsen C, Dijkstra JB, Ponds RW, Wouters EF. [Cognitive impairment in patients with COPD: a review]. Cognitieve beperkingen bij patiënten met COPD: een overzicht. Tijdschr Gerontol Geriatr. 2014;45(1):1-9. Dutch.

11. Lahousse L, Tiemeier H, Ikram MA, Brusselle GG. Chronic obstructive pulmonary disease and cerebrovascular disease: A comprehensive review. Respir Med. 2015;109(11):1371-1380.

12. Kochunov P, Coyle T, Lancaster J, et al. Processing speed is correlated with cerebral health markers in the frontal lobes as quantified by neuroimaging. Neuroimage. 2010;49(2):1190-1199.
13. Marchetta ND, Hurks PP, Krabbendam L, Jolles J. Interference control, working memory, concept shifting, and verbal fluency in adults with attention-deficit/hyperactivity disorder (ADHD). Neuropsychology. 2008; 22(1):74-84.

14. Miller D. Best practices in school neuropsychology: Guidelines for effective practice, assessment, and evidenced-based intervention. Hoboken, NJ: John Wiley and Sons.

15. Leber AB, Turk-Browne NB, Chun MM. Neural predictors of moment-to-moment fluctuations in cognitive flexibility. Proceedings of the National Academy of Sciences of the United States of America. 2008;105(36):13592-13597.

16. Becker BW, Thames AD, Woo E, Castellon SA, Hinkin CH. Longitudinal change in cognitive function and medication adherence in HIV-infected adults. AIDS Behav. 2011;15(8):1888-1894.

17. Brega AG, Grigsby J, Kooken R, Hamman RF, Baxter J. The impact of executive cognitive functioning on rates of smoking cessation in the San Luis Valley Health and Aging Study. Age Ageing. 2008;37(5): 521-525.

18. Lenze EJ, Munin MC, Dew MA, et al. Adverse effects of depression and cognitive impairment on rehabilitation participation and recovery from hip fracture. Int J Geriatr Psychiatry. 2004;19(5):472-478.

19. Cleutjens FA, Wouters EF, Dijkstra JB, et al. The Cognitive-Pulmonary Disease (COgnitive-PD) study: protocol of a longitudinal observational comparative study on neuropsychological functioning of patients with COPD. BMJ open. 2014;4(3):e004495.

20. Global initiative for chronic obstructive lung disease: Pocket guide to COPD diagnosis, management, and prevention. Updated 2015. 2015; http://www.goldcopd.org/uploads/users/files/GOLD_Pocket_2015_ Feb18.pdf. Accessed August 12, 2016.

21. C.B.S. Standaard Beroepenclassificatie 1992 - editie 2001. Den Haag: SDU; 2001.

22. Luteijn F, Barelds DPF. [Groninger Intelligence Test - 2. Manual]. Groninger Intelligentie Test - 2. Handleiding. Amsterdam, the Netherlands: Pearson Assessment and Information BV; 2004. Dutch.

23. Charlson ME, Pompei P, Ales KL, MacKenzie CR. A new method of classifying prognostic comorbidity in longitudinal studies: development and validation. J Chronic Dis. 1987;40(5):373-383.

24. Holland AE, Spruit MA, Troosters T, et al. An official European Respiratory Society/American Thoracic Society technical standard: field walking tests in chronic respiratory disease. Eur Respir J. 2014; 44(6):1428-1446.

25. Zigmond AS, Snaith RP. The hospital anxiety and depression scale. Acta psychiatr Scand. 1983;67(6):361-370.

26. Beck AT, Ward CH, Mendelson M, Mock J, Erbaugh J. An inventory for measuring depression. Arch Gen Psychiatry. 1961;4:561-571.

27. Jolles J, van Boxtel MP, Ponds RW, Metsemakers JF, Houx PJ. De Maastricht Aging Study (MAAS). [The Maastricht aging study (MAAS). The longitudinal perspective of cognitive aging]. Het longitudinaal perspectief van cognitieve veroudering. Tijdschr Gerontol Geriatr. 1998;29(3):120-129. Dutch.

28. Burgmans S, van Boxtel MP, Smeets F, et al. Prefrontal cortex atrophy predicts dementia over a six-year period. Neurobiol Aging. 2009; 30(9): 1413-1419.

29. Singh B, Parsaik AK, Mielke MM, et al. Chronic obstructive pulmonary disease and association with mild cognitive impairment: the Mayo Clinic Study of Aging. Mayo Clinic Proc. 2013;88(11):1222-1230.

30. Cockrell JR, Folstein MF. Mini-Mental State Examination (MMSE). Psychopharmacol Bull. 1988;24(4):689-692.

31. Dulohery MM, Schroeder DR, Benzo RP. Cognitive function and living situation in COPD: is there a relationship with self-management and quality of life? Int $J$ Chron Obstruct Pulmon Dis. 2015(10): 1883-1889.

32. Dal Negro RW, Bonadiman L, Bricolo FP, Tognella S, Turco P. Cognitive dysfunction in severe chronic obstructive pulmonary disease (COPD) with or without Long-Term Oxygen Therapy (LTOT). Multidiscip Respir Med. 2015;10(1):17. 
33. Bucknall CE, Miller G, Lloyd SM, et al. Glasgow supported selfmanagement trial (GSuST) for patients with moderate to severe COPD: randomised controlled trial. BMJ. 2012;344:e1060.

34. Paterniti S, Dufouil C, Bisserbe JC, Alperovitch A. Anxiety, depression, psychotropic drug use and cognitive impairment. Psychol Med. 1999; 29(2):421-428.

35. Vanfleteren LE, Spruit MA, Groenen M, et al. Clusters of comorbidities based on validated objective measurements and systemic inflammation in patients with chronic obstructive pulmonary disease. Am J Respir Crit Care MedS. 2013;187(7):728-735.

36. Cardiogenic brain embolism. Cerebral Embolism Task Force. Arch Neurol. 1986;43(1):71-84.

37. Fan VS, Meek PM. Anxiety, depression, and cognitive impairment in patients with chronic respiratory disease. Clin Chest Med. 2014; 35(2):399-409.

38. Lam RW, Kennedy SH, McLntyre RS, Khullar A. Cognitive dysfunction in major depressive disorder: effects on psychosocial functioning and implications for treatment. Can J Psychiatry. 2014;59(12):649-654.

39. Amado-Boccara I, Gougoulis N, Poirier Littré MF, Galinowski A, Loo H. Effects of antidepressants on cognitive functions: a review. Neurosci Biobehav Rev. 1995;19(3):479-493.

40. Colcombe $\mathrm{S}, \mathrm{Kramer}$ AF. Fitness effects on the cognitive function of older adults: a meta-analytic study. Psychol Sci. 2003;14(2):125-130.
41. Friedman NP, Miyake A, Corley RP, Young SE, Defries JC, Hewitt JK. Not all executive functions are related to intelligence. Psychol Sci. 2006;17(2): $172-179$.

42. Boer LM, Daudey L, Peters JB, Molema J, Prins JB, Vercoulen JH. Assessing the stages of the grieving process in chronic obstructive pulmonary disease (COPD): validation of the Acceptance of Disease and Impairments Questionnaire (ADIQ). Int J Behav Med. 2014;21(3) 561-570.

43. Sulaiman I, Cushen B, Greene G, et al. Objective Assessment of Adherence to Inhalers by COPD Patients. Am J Respir Crit Care Med. 2016 July 13.

44. Finlayson M. Multiple Sclerosis Rehabilitation: From Impairment to Participation. Boca Raton, FL: CRC Press; 2012.

45. Fleming JM, Shum D, Strong J, Lightbody S. Prospective memory rehabilitation for adults with traumatic brain injury: a compensatory training programme. Brain Inj. 2005;19(1):1-10.

46. Merckelbach H, Muris P, Nijman H, de Jong PJ. Self-reported cognitive failures and neurotic symptomatoloy. Pers Indiv Differ. 1996;20(6): 715-724.
International Journal of COPD

\section{Publish your work in this journal}

The International Journal of COPD is an international, peer-reviewed journal of therapeutics and pharmacology focusing on concise rapid reporting of clinical studies and reviews in COPD. Special focus is given to the pathophysiological processes underlying the disease, intervention programs, patient focused education, and self management protocols.

\section{Dovepress}

This journal is indexed on PubMed Central, MedLine and CAS. The manuscript management system is completely online and includes a very quick and fair peer-review system, which is all easy to use. Visit http://www.dovepress.com/testimonials.php to read real quotes from published authors.

Submit your manuscript here: http://www.dovepress.com/international-journal-of-chronic-obstructive-pulmonary-disease-journal 